Pecvnia, 9 (2009), pp. 111-128

\title{
Indicadores de desempeño ambiental en el marco de la ISO 26000 RS $^{*}$
}

Recibido: Octubre 2009

Aceptado: Diciembre 2009

\author{
M. Angela Jimenez \\ Angela.Jimenez@uclm.es \\ Universidad de Castilla La Mancha \\ Fac. de Ciencias Jurídicas y Sociales \\ Cobertizo de San Pedro Mártir, s/n \\ 45071 Toledo (España)
}

La Responsabilidad Social Corporativa (RSC) es un instrumento que ayuda a conciliar objetivos económicos, sociales y medioambientales, a empresas de todos los tamaños, en cooperación con sus interlocutores o partes interesadas, defendiendo valores comunes y aumentando el sentido de la solidaridad y la cohesión, dentro del debate de la globalización, la competitividad y la sostenibilidad.

El impacto de la RSC en la organización se manifiesta en tres dimensiones: económica, social y medioambiental, siendo en esta última, en donde vamos a centrar el trabajo. En el campo
Corporate social responsibility is an instrument that helps to reconcile economic, social and environmental objectives to businesses of all sizes, in cooperation with its partners or stakeholders, to defend common values and increase the sense of solidarity and cohesion, within the debate of globalisation, competitiveness and sustainability.

The impact of CSR in the organization is shown in three dimensions: economic, social and environmental; where we will focus our paper in the latter. In the environmental field,

Este trabajo ha sido presentado como ponencia en el Coloquio Internacional y Consorcio Doctoral sobre Indicadores de Evaluación de la responsabilidad social y medioambiental de las empresas, organizado por ISEOR, Universidad Jean Moulin Lyon 3 y la Academy of Management, cebrado en Lyon los días 8 al 10 de junio del 2009. 
medioambiental es necesario la aplicación de sistemas de gestión que garanticen el cumplimiento de los objetivos dentro del marco de la RSC, bien basados en modelos europeos EMAS o bien según lo establecido por la ISO 14000 y por las primeras aproximaciones de la ISO 26000 RS.

Uno de los instrumentos necesarios para la aplicación de los sistemas de gestión son los indicadores cuantitativos, que nos sirven como cuantificación del desempeño ambiental conseguido por las organizaciones en base a la política establecida. Los indicadores por sí mismos no nos van a proporcionar demasiada información útil, salvo que sean considerados bajo la práctica de otros métodos adicionales como puede ser el análisis DAFO o bien el benchmarking, para seleccionar estrategias en base a la comparativa de la posición de la organización en relación al resto del entorno económico.

Palabras clave: gestión ambiental; normas ISO, indicadores medioambientales. it is necessary the implementation of management systems to ensure the fulfilment of objectives within the framework of the RSC, either based on EMAS European models or on the established ones by the ISO 14000 and by the first approximations of ISO 26000 RS.

One of the necessary instruments for the implementation of management systems are quantitative indicators, which help us as the quantification of environmental performance for organizations based on the established policy. The same indicators will not provide too much information unless they are considered under the practice of other additional methods such as SWOT analysis or benchmarking, to select strategies based on the comparative position of the organization in relation to the rest of the economic environment.

Key words: environmental management, ISO standards, environmental indicators.

\section{LA RESPONSABILIDAD SOCIAL Y EL COMPROMISO AMBIENTAL}

La Responsabilidad Social Corporativa (RSC) se considera, en la actualidad, la filosofía en donde debe enmarcarse el comportamiento empresarial en su conjunto, como estrategia de funcionamiento y supervivencia. Se encuentran diferentes consideraciones y definiciones de lo que se entiende por RSC y, por lo tanto, no existe una única definición ni consideración totalmente aceptada a nivel internacional, lo cual permite múltiples aproximaciones conceptuales, desde diversas ópticas, en base a su criterios generales, objetivos que se pueden alcanzar y elementos a considerar.

En los avances de la Norma ISO 26.000 de Responsabilidad Social (RS), entre mayo del 2006 y noviembre del 2007 en tres reuniones plenarias, se estableció la definición consensuada de la Responsabilidad Social, los siete principios de la norma y los siete temas fundamentales. La definición de la RS quedó de la siguiente forma:

... es la responsabilidad de una organización respecto de los impactos de sus decisiones y actividades en la sociedad 
y el medio ambiente, por medio de un comportamiento transparente y ético que sea:

- Consistente con el desarrollo sostenible, incluyendo el bienestar general de la sociedad

- Considere las expectativas de sus partes interesadas

- Esté en cumplimiento con la legislación aplicable y sea consistente con normas internacionales de comportamiento

- Y esté integrada a través de toda la organización y practicada en sus relaciones

Los siete temas fundamentales son:

- Gobierno organizacional

- Derechos humanos

- Prácticas laborales

- Medio ambiente

- Prácticas operacionales justas

- Temas de consumidores

- Desarrollo social

Concretamente, en el tema medioambiental se incluyen aspectos de esta índole relacionados con las actividades, productos y servicios; promoción del consumo y la producción sostenible; uso sostenible de los recursos; abordar el cambio climático y valoración de servicios ecológicos-ecosistemas.

Con el desarrollo de la ISO RC en conjunción con las ISO 14.000 se puede lograr el establecimiento del medioambiente como requisito de comportamiento socialmente responsable. La simbiosis se podrá producir en el momento de publicación de la definitiva ISO 26.000 que se espera para el segundo semestre del 2010. Si bien, será considerada como una orientación estándar voluntaria y no será considerada como una norma de certificación.

La interconexión entre las comunicaciones europeas y el pronunciamiento internacional de la ISO RS podrá reportar grandes ventajas al conjunto de organizaciones mentalizadas por la actuación socialmente responsable, en donde el medioambiente es un comportamiento primordial. 
Una de las diferentes medidas de desempeño medioambiental de las organizaciones puede cuantificarse mediante un conjunto de indicadores, si bien, no todas las herramientas y modelos de gestión ambiental pueden establecerse en términos numéricos, puesto que el componente cualitativo e intangible es tanto o más importante que el cuantitativo.

En la actualidad, las organizaciones disponen de una potente herramienta de gestión conocida como el Cuadro de Mando Integral o Balanced Score Card, de Kaplan y Norton (1997: 8). La integración de una perspectiva medioambiental que permita cuantificar comportamientos organizaciones puede establecerse como un buen indicador de cumplimientos de objetivos, considerando la integración total de la preocupación y el compromiso actual de las organizaciones. En definitiva, se trata de proporcionar un conjunto de herramientas que nos permitan evaluar y cuantificar el desempeño ambiental en todo tipo de organizaciones. Cada organización podrá aplicar aquel sistema de gestión medioambiental que mejor se adapte a su cultura organizativa y su organización.

En términos generales, podemos encontrar dos grandes modelos de gestión medioambiental, ampliamente admitidos. Se trata del EMS (Environment Management System), basado en la aplicación de las normas ISO 14.000 y el EMAS (Environment Management and Auditing System), conocido como el sistema comunitario de gestión y auditoria medioambiental. Sabiendo que el primero de ellos tiene una aplicación internacional, mientras que el segundo que acopla, fundamentalmente a empresas europeas.

El EMAS tiene como objetivo promover mejoras continuas del comportamiento medioambiental de todas las organizaciones europeas y la difusión de la información pertinente al público y otras partes interesadas. Este sistema se encuentra regulado por el Reglamento (CE) $\mathrm{n}^{\circ}$ 761/2001 del Parlamento Europeo y del Consejo, de 19 de marzo de 2001, por el que se permite que las organizaciones se adhieran con carácter voluntario a un sistema comunitario de gestión y auditoria medioambiental.

De forma resumida, el objetivo de este sistema comunitario de gestión y auditoria es la mejora del comportamiento medioambiental de las organizaciones de todos los sectores mediante:

- El establecimiento y la aplicación, por parte de las organizaciones, de sistemas de gestión medioambiental.

- La evaluación objetiva y periódica de los sistemas. 
- La formación y participación activa del personal de las organizaciones.

- La información al público y otras partes interesadas.

Cada estado miembro establecerá un sistema de acreditación de los verificadores medioambientales independientes y de supervisión de sus actividades.

Este sistema de gestión permite a las empresas alcanzar los siguientes puntos:

- Asegurar un alto nivel de protección del medio ambiente.

- Mejorar continuamente su comportamiento medioambiental.

- Obtener ventajas competitivas.

- Comunicar públicamente sus progresos mediante la obtención de las certificaciones.

Las principales ventajas de implantar el sistema EMAS se pueden resumir en tres grandes bloques:

A) Ahorro de costes derivados de las siguientes actuaciones:

a) Mejora en el control y optimización de consumo de materias primas y energía.

b) Optimización de los costes derivados de la gestión y tratamiento de residuos y emisiones.

c) Reducción de gastos en concepto de transporte, almacenaje y embalaje.

d) Ahorro de costes de trabajos de limpieza y reparación medioambiental derivados de escapes accidentales.

e) Reducción en las primas de seguros por riesgo medioambiental.

f) Negociación de préstamos bancarios en mejores condiciones.

g) Ahorro de sanciones por infracciones.

h) Disminución de riesgo de accidentes y de sus costes derivados.

B) Mejora de la imagen:

a) Las certificaciones suponen una estrategia de marketing.

b) Adopción de una política activa en base a la legislación vigente existente. 
c) Favoreciendo la posibilidad de concesión de permisos y licencias, especialmente con la aprobación de la Directiva 96/61/CE del Consejo, de 24 de abril de 1996, relativa a la prevención y al control integrado de la contaminación.

d) Posibilidad de recibir más ayudas de carácter público para el desarrollo de la iniciativa empresarial.

C) Aumento de la motivación de los empleados:

a) Implicación de la totalidad de los trabajadores del centro en un sistema para conseguir unos objetivos comunes.

b) Aumento de la formación de los trabajadores.

La admisión de la ISO 14001, incorpora un sistema de administración ambiental en todas las fases del proceso de producción, en todos los productos y servicios prestados. Se debe incorporar el concepto medioambiental dentro de los objetivos y metas empresariales.

La adaptación e implantación de una gama de técnicas de gestión medioambiental de una manera sistemática, puede conseguir alcanzar resultados óptimos para todas aquellas partes interesadas, pero no garantizar, en sí mismas, unos resultados medioambientales óptimos. Para lograr objetivos de calidad medioambiental, el sistema de gestión debe utilizar tecnología apropiada y económicamente viable, analizando de forma sistemática los costes de la utilización de la misma (AENOR 1996).

El modelo ISO 14001:2004 tiene un ámbito más amplio que el modelo EMAS, puesto que es internacional, pero no exige ningún tipo de transparencia ambiental. Este sistema plantea grandes ventajas para aquellas empresas que implanten prácticas medioambientales limpias, puesto que aplicarán actuaciones preventivas y mejora de la eficacia de la producción. La adopción de este sistema implicará:

- Garantía de cumplimiento de los requisitos legales.

- Ahorro de costes, debido a la disminución del consumo y de energía.

- Reducción de los riesgos medioambientales.

- Mejora de la competitividad de la empresa.

- Mejora de la imagen de la empresa.

- Mejora de las relaciones tanto internas, con los trabajadores, como externas, clientes, consumidores, etc. 
La adopción de este sistema de gestión medioambiental está certificada por el organismo UNE-EN-ISO.

2. EL PAPEL DE ISO 14.000: 2004 EN LA RESPONSABILIDAD SOCIAL CORPORATIVA EMPRESARIAL. INTEGRACION EN ISO 26000 RS

El conjunto de normas ISO 14.000 se considera como una guía para el desarrollo de la administración del medio ambiente con el objetivo de la estandarización, a nivel internacional, de conceptos como la clasificación y la evaluación del ciclo de vida, conceptos claves para cualquier empresa moderna. Dentro del "compendium" se incorporan un amplio campo de temas, como administración ambiental, auditoría ambiental, desempeño ambiental y otros. Está compuesto por 17 documentos, pero para que cualquier organización quede registrada bajo la ISO 14.000: 2004 sólo debe manifestar su conformidad al documento EMS-ISO 14.001.

El EMS puede ser aceptado para ajustarse a las necesidades de cualquier organización. Su valor está en fomentar un compromiso a favor del progreso ambiental y una mejora constante de la capacidad de la organización para hacer frente a sus responsabilidades y obligaciones ambientales. El EMS permite que las organizaciones cumplan con esas obligaciones ambientales de manera consciente y confiada, tal y como cumplen con sus obligaciones de producción o financieras. De manera resumida podemos establecer que la serie ISO 14.000: 2004 comprende tres grandes campos:

- Objetivos: proporcionar a las organizaciones los elementos de un sistema de administración ambiental, asistencia a las organizaciones que consideren la puesta en práctica o mejora de un sistema de administración ambiental (EMS), incorporando la asesoría necesaria para mejorar el cumplimiento de las funciones medioambientales.

- Estructura de aplicación: modelo de negocios del tipo "planificarhacer-verificar-actuar" y una norma guía separada.

- Contenido: dentro de este compendio de normas se incorporan los elementos de compromiso y responsabilidad de la dirección. Documentación de administración del sistema, control de documentos, control operacional, capacitación, vigilancia y medición, inconformidad y acción correctiva, registros y auditoría. 
De todas ellas, la más relevante en el comportamiento social empresarial está representada en la ISO 14001 que se puede considerar como el documento de especificaciones del sistema de administración de la serie ISO 14000. Incorpora aquellos elementos que deben ser cumplidos para que una organización pueda conseguir el registro o certificación de esta norma. Un sistema de administración ambiental es la parte del sistema de administración general que incluye estructura, planificación, actividades, responsabilidades, prácticas, procedimientos, procesos y recursos de una organización para desarrollar, poner en práctica, alcanzar, revisar y mantener la política ambiental. Podemos considerarla como el requisito para el desarrollo del tema medioambiental propuesto por la ISO 26000 RS, como instrumento de implantar la preocupación medioambiental en las organizaciones.

La ISO 14001 se puede considerar como un indicador de la aceptación y compromiso, por parte de la organización, de la protección del medio ambiente, dentro del comportamiento socialmente responsable de las organizaciones.

La aportación que proporciona la aceptación de esta norma para aquellas empresas socialmente responsables, los podemos resumir en los siguientes:

- Autoevaluación inicial de Gestión ambiental.

- Compromiso y política. Creación de la cultura medioambiental.

- Planificación de la política ambiental, para lo cual necesita:

- Identificación y registro de los aspectos medioambientales y evaluación de los impactos ambientales.

- Requisitos legales.

- Criterio de comportamiento interno.

- Establecer objetivos y metas ambientales.

- Desarrollo de un programa de gestión ambiental.

- Implementación y desarrollo de las capacidades y mecanismos de la política, objetivos y metas ambientales.

- Medición y evaluación para un seguimiento de la política y realización de un feed-back de la misma.

- Revisión, mejoramiento y comunicación para informar a las partes involucradas de los logros ambientales obtenidos.

Todas estas variables no pueden considerarse elementos independientes; se establecen relaciones entre ellas y se considera 
primordial y necesario que todas se incluyan para desarrollar la actividad de la unidad económica, con el objetivo último de mantener o incrementar la posición competitiva de una empresa. En la actualidad, se desarrollan ante la creación del cuadro de mando medioambiental y las memorias de sostenibilidad de las organizaciones.

Enmarcado en la Política de Calidad Total de la empresa, el sistema de gestión medioambiental completa el proceso de mejora continua, entendiendo como tal el proceso de intensificación del sistema de gestión medioambiental para la obtención de mejoras en el comportamiento de este campo, de manera global, de acuerdo con la política medioambiental de la organización, no siendo necesario aplicarse a todas las áreas de la actividad de la empresa de una forma simultánea.

Dentro de la gestión de este sistema, los aspectos más significativos asociados a las actividades de las unidades operativas, encontramos:

- Emisiones atmosféricas.

- Vertidos al agua.

- Gestión de residuos.

- Contaminación del suelo.

- Utilización de la materia prima y recursos naturales.

- Otras cuestiones medioambientales que afecten a la comunidad.

La gestión medioambiental trata de identificar todos los aspectos significativos asociados a las actividades, productos o servicios, no implicando la evaluación de forma unitaria de cada output final. Cada organización se encargará de agrupar por actividades, por lotes de productos o por tipología de servicios prestados según las características inherentes a ella misma. una serie de etapas:

En términos generales, todo proceso de gestión se divide en

a) Planificación y presupuestación.

b) Aplicación de procedimientos.

c) Evaluación de datos predeterminados.

d) Comparación con datos reales.

e) Procesos de control y auditoría.

f) Análisis de resultados y medidas correctivas. 
Existe un proceso de retroalimentación o feed-back encargado de reconducir al sistema para la consecución de objetivos y metas planificados.

3. EL PAPEL DE LOS INDICADORES EN LA GESTIÓN MEDIOAMBIENTAL COMO MEDIDA DEL DESEMPEÑO AMBIENTAL

En base a la normativa internacional, ISO 14001 e ISO 14031, y en relación con los primeros desarrollos de la ISO 26000 RS, el desempeño ambiental puede interpretarse como "Resultados medibles del sistema de gestión ambiental, relativos al control de los aspectos ambientales de la organización, basados en su política ambiental, objetivos y metas"; "Resultados de la gestión ambiental de la organización en relación con los aspectos ambientales".

El problema que se plantea a la hora de analizar los datos derivados del análisis de la gestión ambiental es la posibilidad de cuantificar los resultados. No debemos olvidar, que no es posible realizar una cuantificación totalmente fiable de aspectos, cuyo fundamento se basa en la filosofía de comportamiento social de las organizaciones, en donde, se debe incorporar un aspecto subjetivo relevante.

La aplicación de ratios para la evaluación y análisis de la gestión de las organizaciones ha tomado una mayor relevancia desde la implantación del Cuadro de Mando y Balanced Score Card, como herramienta en el proceso de toma de decisiones, bajo la restricción de que no todo comportamiento es cuantificable ni extrapolable en términos matemáticos. De ahí la proposición inicial de incorporar la perspectiva medioambiental en la elaboración del los Cuadros de Mando de empresas consideradas como socialmente responsables.

En términos generales, los indicadores medioambientales se encargan de resumir datos extensos en información clave significativa y comparable, con el fin de presentar el comportamiento medioambiental de una empresa de manera exhaustiva y cuantificable, como medida de gestión de la política medioambiental.

Si bien, dentro del conjunto de indicadores que se pueden definir, se debe diferenciar entre los indicadores en términos absolutos, que no indicarán el consumo de recursos y sus emisiones y los elaborados en términos relativos, que se encargarán de considerar la eficiencia de la producción, para el análisis de la mejora. 
La metodología para la implantación de los mismos la podemos resumir en los siguientes pasos:

Gráfico 1: Metodología de implantación de un sistema de elaboración de ratios medioambientales

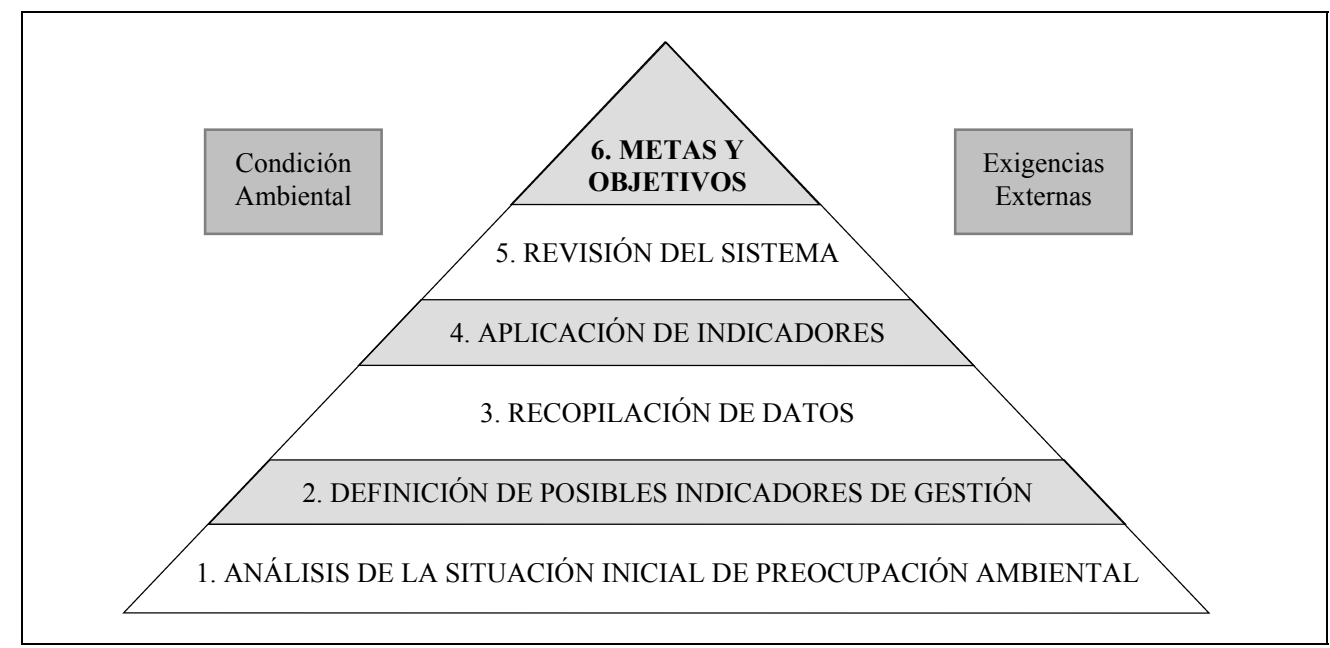

En términos generales, cuando una organización se implica con el medioambiente y decide aplicar un EMAS (Sistema Europeo de Certificación en Gestión Medioambiental) o bien un EMS ISO 14000 (sistema de certificación internacional), necesita plantearse una serie de preguntas, en relación con el conjunto de indicadores, demandados por la sociedad, que le permita definir qué información demandan los usuarios y compromisarios externos e internos en la aplicación de esta política de gestión empresarial y cómo se están alcanzando las ventajas competitivas que suponen.

En estos términos, tendremos que cuantificar aspectos relacionados con los siguientes puntos:

- Ahorro de costes: mejora en el control de materias primas y energía; mejor posición para obtener préstamos y subvenciones; optimización de los costes de residuos y emisiones; reducción de los riesgos de accidentes y los costes de las reparaciones por daños al medio ambiente; mejoras en transportes, envasados, etc.

- Ventajas de competitividad: buena imagen de la empresa; relaciones con los agentes externos; aumento de motivación de los empleados.

- Cumplimiento de la legislación vigente. 
En estos términos, considerando diversos informes de la Comisión Europea, para el desarrollo cuantitativo de las ventajas expuestas anteriormente, se deben considerar cuatro grandes bloques de indicadores, que son:

- Indicadores que pueden elaborarse, porque las organizaciones disponen de datos y son fiables.

- Indicadores que puede elaborarse de forma parcial, puesto que los datos se manifiestan incompletos y no se encuentran actualizados.

- Indicadores que no se podrán elaborar ni el futuro, puesto que aun con la existencia de datos no se consideran fiables para la realización de un análisis exhaustivo.

- Indicadores, que aun contando con datos disponibles no resultan suficientemente significativos y requieren de apoyos metodológicos diferentes.

Si bien la Comunicación de la Comisión de 8 de octubre de 2003 establece una lista reducida de los 14 indicadores estructurales medioambientales, así como aquellos que deberán elaborarse para un futuro, a nivel de países miembros y afines, nos centraremos en los que deben definir las organizaciones, para garantizar la demanda de información de los usuarios y que deben ser considerados en sus memorias de sostenibilidad; debiendo reconocer que en base a su actividad, la variante de los mismos puede ser significativa.

En términos generales, los indicadores medioambientales de la empresa se pueden clasificar en tres grandes bloques, y de cada uno de ellos, se reconocen un conjunto adicional de ellos:

1. Indicadores de Comportamiento Medioambiental

2. Indicadores de Gestión Ambiental

3. Indicadores de Situación Ambiental

4. DESARROLLO DE INDICADORES MEDIOAMBIENTALES EN BASE A LA NORMATIVA INTERNACIONAL ISO 26000 RS Y NORMATIVA EUROPEA

El desglose podría elaborarse de la siguiente forma, en base a la consideración de la perspectiva medioambiental del cuadro de mando integral planteado por Kaplan y Norton. 


\section{Indicadores de Comportamiento Medioambiental:}

\begin{tabular}{|c|c|}
\hline \multicolumn{2}{|c|}{ a. Indicadores de Materiales y Energía } \\
\hline $\begin{array}{l}\text { Indicadores de consumo: factores utilizados } \\
\text { por la organización para el desarrollo del } \\
\text { compromiso medioambiental }\end{array}$ & $\begin{array}{l}\text { - Materiales: consumo de materiales recuperados por } \\
\text { unidad producida; consumo de materiales recuperados } \\
\text { por kg de consumo total. } \\
\text { - Energía: Consumo absoluto de energía; consumo de } \\
\text { energía por unidad producida; consumo de energía } \\
\text { renovable por unidad producida; porcentaje de consumo } \\
\text { de energía de renovable con relación al consumo total. } \\
\text { - Agua: consumo de agua potable por unidad producida. } \\
\text { Consumos por unidades de tiempo. }\end{array}$ \\
\hline $\begin{array}{l}\text { Indicadores de outputs: análisis de los outputs } \\
\text { producidos en base a la gestión ambiental }\end{array}$ & $\begin{array}{l}\text { - Producción de Residuos peligrosos: en términos } \\
\text { absolutos; por unidad producida. } \\
\text { - Emisiones Atmosféricas (ozono, partículas y dióxido } \\
\text { de azufre). Tasa de emisión de gases de efecto } \\
\text { invernadero. } \\
\text { - Aguas Residuales: metro cúbico de agua por medida } \\
\text { de producción. } \\
\text { - Productos. } \\
\text { - Vertidos de sustancias contaminantes (fertilizantes, } \\
\text { materias orgánicas, productos químicos). }\end{array}$ \\
\hline \multicolumn{2}{|c|}{ b. Indicadores de Infraestructura y Transporte } \\
\hline $\begin{array}{l}\text { Indicadores de infraestructura: análisis de } \\
\text { comportamiento externo e interno ligado } \\
\text { con factores condicionantes de la estructura } \\
\text { productiva y de prestación de servicios de la } \\
\text { empresa. }\end{array}$ & $\begin{array}{l}\text { - Consumo de sustancias químicas tóxicas. } \\
\text { - Productividad de los recursos. } \\
\text { - Tasa de reciclaje de los materiales (papel, cartón y } \\
\text { vidrio). } \\
\text { - Generación de residuos peligrosos. } \\
\text { - Gastos en tecnologías de información. } \\
\text { - Economía del Conocimiento: tasa de coste de formación } \\
\text { en materia medioambiental por empleado. }\end{array}$ \\
\hline $\begin{array}{l}\text { Indicadores de Transporte: medidas } \\
\text { cuantitativas centradas en el análisis de la } \\
\text { función de externa de relación de la empresa } \\
\text { con usuarios. Estos indicadores de transporte } \\
\text { podrían derivarse de la política de just-in- } \\
\text { time, con el objetivo de la reducción de } \\
\text { costes de aprovisionamiento, en el amplio } \\
\text { sentido. }\end{array}$ & $\begin{array}{l}\text { - Consumo energético según el modo de transporte. } \\
\text { - Distribución modal de los transportes (dependencia } \\
\text { del transporte por carretera para las mercancías). } \\
\text { - Inversiones en infraestructuras por transporte. } \\
\text { - Volúmenes del transporte de carga. } \\
\text { - Ahorro de costes de carburantes: por utilización de } \\
\text { aquellos medioambientales. }\end{array}$ \\
\hline
\end{tabular}

En relación a la tasa de reciclado de los materiales, papel, cartón y vidrio, se debe considerar que por reciclado se entiende cualquier reutilización de un material en un proceso de producción o consumo que lo retire del flujo de residuos. Y en este caso, atendiendo al Informe de la Comisión al Consejo (COM 2002) 524 final "Análisis de la lista abierta de 


\section{indicadores de cabecera medioambientales", se puede a su vez subdividir en dos elementos:}

1. Porcentaje de reciclado de vidrio

2. Porcentaje de reciclado de papel y cartón

Si bien, otro indicador alternativo podría ser la relación entre la cantidad de materiales de desecho recogidos para reciclarlos y el volumen total de materiales de desecho generado.

\section{Indicadores de Gestión Ambiental (independientemente} del modelo de gestión medioambiental aplicado)

\begin{tabular}{|c|c|}
\hline $\begin{array}{l}\text { Indicadores del sistema: expresión cuantitativa } \\
\text { de la aplicación del sistema de gestión } \\
\text { ambiental desarrollado por la organización. } \\
\text { Análisis cuantitativo de desempeño ambiental } \\
\text { en función del modelo ISO } 14.000 \text { ó EMAS }\end{array}$ & $\begin{array}{l}\text { - Implantación del sistema ambiental: resultados de las } \\
\text { auditorias medioambientales; costes de la auditoria } \\
\text { por persona. Coste de la auditoria por línea de } \\
\text { producto; por sector de mercado; o por planta. } \\
\text { - Aspectos Legales: cumplimiento de legislación vigente; } \\
\text { porcentaje de sanciones por ejercicio. } \\
\text { - Costes ambientales: en base al análisis de costes de } \\
\text { calidad. Costes de prevención, coste de evaluación y } \\
\text { costes de fallos. }\end{array}$ \\
\hline $\begin{array}{l}\text { Indicadores de funcionamiento: expresión } \\
\text { cuantitativa del desarrollo de la política } \\
\text { ambiental, como puesta en práctica del } \\
\text { sistema de gestión elegido }\end{array}$ & $\begin{array}{l}\text { - Formación del personal: unidades monetarias } \\
\text { invertidas por recurso humano; unidades económicas } \\
\text { por ejercicio. } \\
\text { - Seguridad e higiene: niveles de exposición a } \\
\text { contaminantes por persona; medidas de exposición } \\
\text { acústica por persona. } \\
\text { - Compras de factores; porcentaje de factores empleados } \\
\text { sostenibles por producto obtenido; porcentaje de } \\
\text { factores reciclables empleados por producto obtenido. } \\
\text { - Relaciones externas: coste por producto de } \\
\text { asesoramiento en materia ambiental; coste por } \\
\text { persona de asesoramiento en materia ambiental; coste } \\
\text { por planta; coste por línea de producto; coste por } \\
\text { sección de mercado. }\end{array}$ \\
\hline $\begin{array}{l}\text { Indicadores de la Situación ambiental: } \\
\text { expresión cuantitativa del compromiso } \\
\text { ambiental de la organización con el entorno, } \\
\text { tanto propio de trabajo como a nivel general } \\
\text { de la sociedad. }\end{array}$ & $\begin{array}{l}\text { - Indicadores de agua: medidas de pureza de agua de } \\
\text { lagos cercanos a la empresa; contaminación del agua } \\
\text { de lugares cercanos; utilización de agua en el proceso } \\
\text { de actividad; concentración de nitratos y fosfatos en } \\
\text { acuíferos cercanos a la empresa. } \\
\text { - Indicadores de Suelo: concentración de elementos } \\
\text { contaminantes por metro cuadrado; porcentaje de } \\
\text { recuperación de suelo para la agricultura; tasa de } \\
\text { enriquecimiento de suelo por metro cuadrado. } \\
\text { - Indicadores de aire: pureza de aire por metro cúbico } \\
\text { dentro de la organización; porcentaje de humedad }\end{array}$ \\
\hline
\end{tabular}




\begin{tabular}{|l|l|}
\hline & $\begin{array}{l}\text { necesaria para la buena respiración; niveles de partidas } \\
\text { contaminantes en el aire del centro de trabajo. } \\
- \text { Indicadores de Flora y Fauna: porcentajes de } \\
\text { recuperación de flora; participación en proyectos } \\
\text { sociales de recuperación de flora y fauna; tasa de } \\
\text { beneficios destinados a proyectos públicos o privados } \\
\text { ambientales. }\end{array}$ \\
\hline
\end{tabular}

La definición de los indicadores y el análisis de los mismos dependerá del tipo de organización en la que se implante la gestión ambiental. En el caso de pequeñas y medianas empresas, el comportamiento se focalizará en los indicadores del comportamiento medioambiental. Si hablamos de grandes empresas, la focalización del análisis cuantitativo tenderá hacia los indicadores de comportamiento y de gestión ambiental.

\section{CONSIDERACIONES FINALES}

Evidentemente, el análisis de indicadores no tiene validez por sí mismo, salvo por comparación con la situación en periodos previos, o bien, mediante la aplicación del benchmarking con otras empresas del sector, para analizar la evaluación comparativa. Esta posición nos servirá para la realización del análisis SWOT y detectar los puntos débiles y potenciales de mejora. El Análisis FODA o Análisis DAFO (SWOT - Strengths, Weaknesses, Opportunities, Threats) es una metodología de estudio de la situación competitiva de una empresa en su mercado y de las características internas de la misma, a efectos de determinar sus Fortalezas, Oportunidades, Debilidades y Amenazas; las debilidades y fortalezas son internas a la empresa; las amenazas y oportunidades son externas, y se presentan en su entorno. Durante la etapa de planificación estratégica y a partir del análisis DAFO se debe poder contestar cada una de las siguientes preguntas:

- ¿Cómo se puede aprovechar cada fortaleza medioambiental?

- ¿Cómo se puede explotar cada oportunidad de gestión medioambiental?

- ¿Cómo se puede detener cada debilidad en la aplicación de la gestión medioambiental?

- ¿Cómo se puede defender de cada amenaza externa para la aplicación de la política y gestión medioambiental? 
A su vez, este análisis puede complementarse con el ciclo Deming, también conocido como ciclo PDSA o rueda de Deming, de la Calidad Total (Planear, Hacer, Verificar y Actuar), que permite alcanzar la mejora continua.

En la actividad de Planificación, se seleccionarán el conjunto de indicadores medioambientales a aplicar. La acción, se centrará en la recolección de datos, la conversión de los mismos en información a aplicar, el análisis de la información y la obtención de resultados. Este conjunto de datos se elaborarán mediante un informe, que se comunicará a la dirección competente. Posteriormente, pasamos a las actuaciones de verificación y actuación, que supondrán la revisión del sistema y las mejoras necesarias para alcanzar la planificación desarrollada y alcanzar el objetivo de excelencia.

El conjunto de indicadores se pueden realizar en tres ámbitos diferentes, o bien de forma general en el conjunto global de la empresa, o bien por plantas o divisiones o bien por centros individuales, dependiendo de la información que pueda obtenerse y elaborarse, en términos generales. Los indicadores de gestión ambiental, deberían elaborarse en términos globales, pues la empresa u organización hay que considerarla como un todo y como un elemento interrelacionado con la sociedad y, además, hablamos de una filosofía de comportamiento que incide en la gestión global, que conduce a la excelencia. Mientras que sería más fácil si los indicadores de comportamiento ambiental se elaboraran por plantas o divisiones, lo que nos permitiría analizar la eficacia con el objetivo de la consecución global de las metas y los objetivos medioambientales de la organización.

En definitiva, y a modo de conclusión, cualquier conjunto de indicadores deben cumplir un conjunto de requisitos que permitan obtener una información útil para el proceso de toma de decisiones. Entre ellas podemos encontrar:

- Comparabilidad: lo cual nos permitirá realizar comparaciones o bien con referencia a periodos anteriores, o bien en referencia al comportamiento de otro conjunto de empresa.

- Deben estar dirigidos a alcanzar los objetivos y metas propuestas y la excelencia.

- Deben establecerse con el objetivo de continuidad. Es decir, no se debe modificar constantemente el conjunto de indicadores, puesto 
que carecerían de la comparabilidad. En este caso no se habla de complementación.

- Deben estar elaborados con una periodicidad que nos permita analizar el comportamiento y la gestión ambiental de manera razonable, objetiva, relevante y útil.

- Claridad en la elaboración del indicador y en información derivada el mismo, para que sea fácilmente entendida por todo el conjunto de usuarios.

\section{BIBLIOGRAFÍA}

AENOR: UNE-EN ISO 14001 (1996) Sistemas de gestión medioambiental. Especificaciones y directrices para su utilización.

CASCIO, J. (1997) Guía ISO 14.000. Las Nuevas Normas internacionales para la administración ambiental. México: Mc Graw Hill.

-, G. WOODSIDE y P. MITCHELL (1996) Guía ISO 14.000. México: Mc Graw Hill.

Comunicación de la Comisión COM (2001) "Desarrollo sostenible en Europa para un mundo mejor: estrategia de la Unión Europea para un desarrollo sostenible". Bruselas. 264 final.

Comunicación de la Comisión COM (2002) "Análisis de la lista abierta de indicadores de cabecera medioambiental". Bruselas. 524 final.

Comunicación de la Comisión COM (2005) "Revisión de la Estrategia para un desarrollo sostenible". Bruselas. 658 final.

Comunicación de la Comisión COM (2007) "Informe provisional sobre la Estrategia de Desarrollo Sostenible 2007". Bruselas. 642 final.

D'ANGELO, S. (1995) L'ambiente fra sanzioni e strumenti volontari: le responsabilità penali ed administrative dell'impresa a confronto con Ecolabel ed Ecoaudit. Milano: Pirola.

Delogu, B.; M. Dubini ed P. GIUIUZza (1996) Gestire l'ambiente. Milano: Pirola.

Douglas Muschett, F. (1998) Principios del Desarrollo Sostenible. Madrid: AENOR.

INTERNATIONAL STANDARD ORGANIZATION: ISO 14001. Section 3.5. Genève. 1996.

ISO Responsabilidad Social [www.vincular.org/index/base0.php]. 
JIMÉNEZ MONTAÑÉS, M.A. (1999) "Los nuevos costes empresariales ante el compromiso medioambiental de las organizaciones (ISO 14000)", VI Congreso Internacional de Custos, Braga, Portugal: 17-20 septiembre.

- (2001) "Incidencia de los Nuevos Costes Empresariales, Calidad y Medioambiente en el modelo de toma de decisiones a $\mathrm{c} / \mathrm{p}$. Caso particular C-V-B", VII Congreso del Instituto Internacional de Costos y II Congreso de ACODI, León, España: 4-6 julio.

KAPLAN, R.S. y D. NORTON (1997) Cuadro de Mando Integral (The Balanced Scorecard). Barcelona: Gestion 2000.

OHMAE, K. (1982) The Mind of the Strategist: The Art of Japanese Business. New York: Mc Graw-Hill.

Real Decreto 833/1988, de 20 de julio, por el que se aprueba el reglamento para la ejecución de la Ley 20/1986, de 14 de mayo.

Reglamento (CEE) 1836/93 del Consejo, de 29 de junio de 1993, por el que se permite que las empresas del sector industrial se adhieran con carácter voluntario a un sistema comunitario de gestión y auditoría medioambientales. DOCE 168/L, de 10-07-93.

RITCHIE, I. (1998) A Guide to the Implementation of the ISO 14000 Series on Environmental Management. Upper Saddle River, NJ: Prentice Hall.

Stakeholders Consensus Enables ISO 26000 on Social Responsibility to Move Up in Development Status [www.isotc.iso.org/pressrelease.htm]. 